\title{
Living with Ulcerative Colitis in Japan: Biologic Persistence and Health-Care Resource Use
}

\author{
Danielle Bargo $^{a} \quad$ Theo Tritton $^{b}$ Joseph C. Cappelleric Marco DiBonaventura ${ }^{c}$ \\ Timothy W.Smith $^{c}$ Takanori Tsuchiya ${ }^{d}$ Sean Gardiner ${ }^{a} \quad$ Irene Modesto $^{a}$ \\ Tim Holbrook $^{b}$ Daniel Bluff ${ }^{b}$ Taku Kobayashi ${ }^{\mathrm{e}}$
}

aPfizer Inc, New York, NY, USA; ${ }^{\text {b} A d e l p h i ~ R e a l ~ W o r l d, ~ M a n c h e s t e r, ~ U K ; ~ ' P f i z e r ~ I n c, ~ G r o t o n, ~ C T, ~ U S A ; ~ d P f i z e r ~ I n c, ~ T o k y o, ~}$

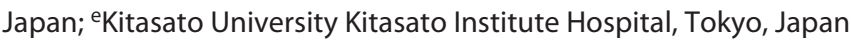

\section{Keywords}

Adherence - Tumor necrosis factor inhibitor - Health-care resource utilization · Persistence - Ulcerative colitis

\begin{abstract}
Objective: The aim of the study was to improve understanding of adherence and persistence to biologics, and their association with health-care resource utilization (HCRU), in Japanese patients with moderate to severe ulcerative colitis (UC). Methods: Data were from Medical Data Vision, a secondary care administrative database. A retrospective, longitudinal cohort analysis was conducted of data from UC patients initiating biologic therapy between August 2013 and July 2016. Data collected for 2 years prior (baseline) and 2 years after (follow-up) the index date were evaluated. Patients completing biologic induction were identified, and adherence/persistence to biologic therapy calculated. HCRU, steroid, and immunosuppressant use during baseline and follow-up were assessed. Biologic switching during the follow-up was evaluated. Descriptive statistics (e.g., means and proportions) were obtained and inferential analyses (from Student's $t$ tests, Fisher's exact tests, $X^{2}$ tests, the Cox
\end{abstract}

proportional hazard model, and negative binomial regression) were performed. Results: The analysis included 649 patients (adalimumab: 265; infliximab: 384). Biologic induction was completed by $80 \%$ of patients. Adherence to adalimumab was higher than that to infliximab $(p<0.001)$. Persistence at $6,12,18$, and 24 months was higher with infliximab than with adalimumab $(p<0.05)$. Overall, gastroenterology outpatient visits increased, and hospitalization frequency and duration decreased, from baseline to follow-up. UC-related hospitalizations were fewer and shorter, and endoscopies fewer, in persistent than in nonpersistent patients, although persistent patients made more outpatient visits than nonpersistent patients. Hospitalization duration was lower in persistent than nonpersistent patients. Approximately $50 \%$ of patients received an immunosuppressant during biologic therapy; $5 \%$ received a concomitant steroid during biologic therapy. Overall, $17 \%$ and $3 \%$ of patients, respectively, received 2 nd line and 3rd line biologics. Conclusions: Poor biologic persistence was associated with increased nonmedication-associated HCRU. Effective treatments with high persistence levels and limited associated HCRU are needed in UC.

(c) 2021 The Author(s)

Published by S. Karger AG, Basel
C 2021 The Author(s).

Published by S. Karger AG, Basel

This is an Open Access article licensed under the Creative Commons Attribution-NonCommercial-4.0 International License (CC BY-NC) (http://www.karger.com/Services/OpenAccessLicense), applicable to the online version of the article only. Usage and distribution for commercial purposes requires written permission.
Correspondence to:

Danielle Bargo, dnbargo@gmail.com 


\section{Introduction}

Ulcerative colitis (UC) is a chronic inflammatory bowel disease, in which there is inflammation of a continuous section of the colonic mucosa from the rectum extending proximally for variable distances [1-3]. UC is characterized by periods of remission and relapse, with patients who initially present with UC affecting a limited section of the colon often progressing to more extensive disease [4]. The most common presenting symptoms are bloody diarrhea, abdominal pain, urgency, and tenesmus $[1,5]$.

The prevalence of $\mathrm{UC}$ ranges from 2.4 to 505 cases per 100,000 people across different regions of the world [6]. The prevalence in Japan has been reported to be $18.1 / 100,000$ in 1991 [7], 63.6/100,000 in 2005 [8], and $172.9 / 100,000$ in 2014 [9], in line with a reported increase in the incidence of UC $[6,10]$.

Pharmacological interventions available for UC include aminosalicylates (5-ASA), corticosteroids, immunosuppressants (IMs), and biologics. Surgery is an option for nonresponsive patients with severe disease $[11,12]$. Japanese, European, and US guidelines for the management of UC recommend that disease extent, severity, and current activity are taken into account when selecting treatment, although Japanese guidelines do not specify a treatment target [13-16]. For the induction of remission in moderate-to-severe UC, systemic steroids are the most common first-line treatment; biologics are an option for second-line therapy; intravenous steroids are usual given in acute severe UC; intravenous IM therapy or the biologic infliximab may be used as rescue therapy if there is insufficient response to first-line treatment. For patients in remission, maintenance treatment options include oral 5-ASA, oral IMs, or biologics if these have been used in inducing remission. Biologics approved for treatment of UC in Japan include infliximab, adalimumab, golimum$a b$, and vedolizumab $[17,18]$, although golimumab was only approved in March 2017 and vedolizumab in July $2018[19,20]$.

Although biologics are effective in a high proportion of patients with UC, a substantial proportion of patients fail to reach treatment targets $[18,21]$, with $\sim 50 \%$ of patients achieving clinical response, and fewer than $25 \%$ achieving remission [17]. Furthermore, the development of anti-drug antibodies can lead to a secondary loss of response; a systematic review of international studies reported anti-drug antibodies in up to $41 \%$ of patients with UC receiving biologics [22].

A study of Japanese claims data reported that 55\% of patients with UC persisted to biologic medication

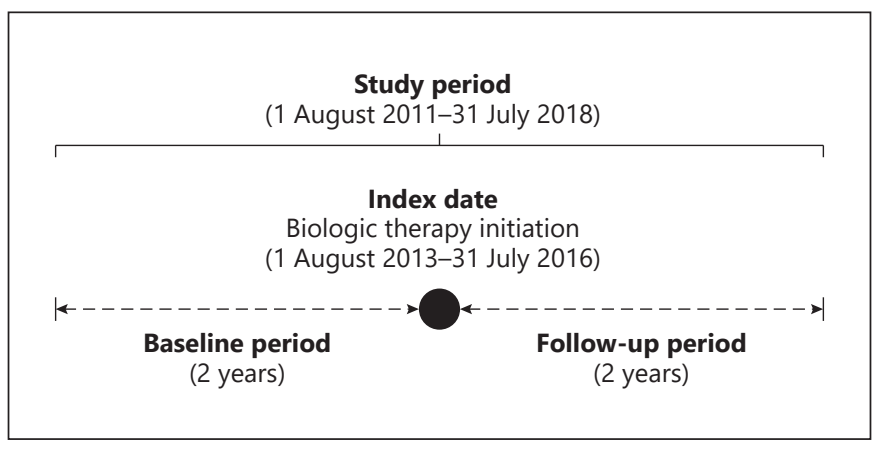

Fig. 1. Study design.

throughout the maintenance period, with persistence to infliximab slightly better than adalimumab [23]. There is also little known about the impact of suboptimal adherence and persistence to biologics, which are known to be associated with suboptimal outcomes for 5-ASA [24, 25].

The aim of this study was to describe adherence and persistence to infliximab and adalimumab in Japanese patients with moderate-to-severe UC, as these are indicators of treatment response to biologics. A better understanding of these variables may inform management of patients with UC. This study also assessed the association of adherence and persistence to these biologics; the use of steroids and IMs in combination with biologic therapy; the extent to which patients had changes in the biologic agent received; and potential predictors of biologic therapy persistence and UC-related hospitalizations.

\section{Materials and Methods}

\section{Study Design}

Data were obtained from Medical Data Vision (MDV), a secondary care administrative database in Japan [26, 27]. MDV holds data from $22 \%$ of all acute care hospitals in Japan. It should be noted that although "acute care" is specified, data are available on all types of care provided by these institutions. Information on $>25$ million individuals (outpatient and inpatient) is held. MDV includes data from 2008 onward.

A retrospective, longitudinal cohort analysis was conducted using data from patients with UC initiating biologic therapy between August 1, 2013 and July 31, 2016 - the index date (Fig. 1). As only infliximab and adalimumab were approved for use in UC in Japan during this period, the analysis included these 2 biologics. Data collected during the 2 years prior to the index date (baseline period) and the 2 years after the index date (follow-up period) were evaluated. This study is one of two studies conducted to describe real-world outcomes in patients with UC, and the other to describe health-care resource utilization (HCRU) and treatment patterns among patients with UC in Germany [28] (shown in Fig. 1). 


\section{Patients}

Eligible patients were 18 years or older on the index date, had one confirmed diagnosis code for UC (International Classification of Diseases, 10th Revision [ICD-10] code K51.X) in the 2 years prior to or including the index date, had an outpatient prescription or inpatient administration of either adalimumab or infliximab between August 2013 and July 2016, and had data available in MDV throughout the baseline and follow-up periods. Patients were excluded from the analysis if they had a prescription or administration of $\geq 2$ of either specified biologic therapy on the index date, one or more prescription(s) or administration(s) of one of the specified biologic therapies in the baseline period, or one or more confirmed ICD-10 diagnosis code for Crohn's disease (K50.X) in the baseline or follow-up period.

\section{Outcomes}

Patient demographics and clinical characteristics (including extent of disease and Charlson Comorbidity Index (CCI) at end of baseline) were reported [29]. Patients completing biologic induction were identified using standard induction periods: adalimumab: a prescription after 21 days; infliximab: a prescription after 56 days. When the induction period for adalimumab was defined as 35 days, an equal number of patients were shown to reach maintenance therapy.

Adherence to the indexed biologic therapy during the maintenance period was measured as the proportion of days covered (PDC: the number of days in period covered by prescription/the number of days in period) [30]. The number of days' supply of adalimumab was estimated using the amount prescribed and a defined daily dose (DDD) of $2.86 \mathrm{mg}$ ( $40 \mathrm{mg}$ every other week), as defined by the World Health Organization ATC/DDD index. For infliximab, a dosage of $6.25 \mathrm{mg} / \mathrm{kg}$ was used to better reflect clinical practice $(5 \mathrm{mg} / \mathrm{kg}$ every 8 weeks for a $70-\mathrm{kg}$ patient corresponds to $6.25 \mathrm{mg} / \mathrm{kg}$ daily). PDC was calculated until discontinuation or end of the follow-up, with discontinuation defined as a gap of $\geq 60$ days between fills/infusions. Patients were categorized as adherent (PDC $\geq 80 \%$ ) or nonadherent (PDC $<80 \%$ ).

Persistence to the indexed biologic therapy was defined as the time from the index date to discontinuation, with discontinuation defined as a gap of $\geq 60$ days between fills/infusions, and the number of days' supply of biologic medication estimated using the amount prescribed and recommended doses, as described before. The proportions of patients persisting at $6,12,18$, and 24 months were calculated. Sensitivity analyses were performed to explore persistence rates with discontinuation defined as a gap of $\geq 30$ days and $\geq 90$ days between fills/infusions.

HCRU was assessed in the baseline and follow-up periods. Resources considered were therapy for UC (biologics, 5-ASA, steroids and IMs), outpatient appointments, hospital stays, visits to the emergency room, and any UC-related procedures or surgeries. Resource uses with an associated diagnosis code for UC were considered "UCrelated." These were reported, and HCRU was compared between persistent and nonpersistent patients at 6,12, and 18 months.

Assessment of the use of steroids and IMs in the baseline and follow-up periods included consideration of combination treatment strategies (biologic + steroid, biologic + IM, biologic + steroid + IM). To account for steroid tapering, patients were required to have received a steroid prescription 14 weeks or more after initiation of biologic therapy to be considered to have received biologic + steroid therapy.
Any overlap in biologic and IM prescriptions was considered to indicate biologic + IM therapy. Biologic + IM use was further split into those who initiated IM within 3 months of initiating biologic therapy and those who initiated IM $>3$ months after initiating biologic therapy. The proportions of patients switching to a different tumor necrosis factor inhibitor (TNFi) during the follow-up period for treatment beyond first-line (referred to as cycling) were calculated. In addition to infliximab and adalimumab, golimumab was included as second- or third-line biologic therapy.

\section{Analysis}

All outcomes were analyzed descriptively and reported using frequencies and percentages for categorical variables, and counts, means, medians, standard deviations, and interquartile ranges (IQR) for continuous variables. For unpaired statistical comparisons between the 2 independent groups (adalimumab vs. infliximab), we have applied the following tests: Student's $t$ test for difference in outcome means, Fisher's exact test (for $2 \times 2$ cross-classification tables when an expected cell count was $<5$ ), and $\chi^{2}$ test (for cross-classification tables when each expected cell count is 5 or greater) for binary or multinomial outcomes [31, 32].

Predictors for patients persisting with their indexed therapy in the 2-year follow-up period were examined using a Cox proportional hazards model [32], with potential predictors including indexed biologic, steroid and IM use, sex, baseline CCI, and years since diagnosed with UC ( $\leq 1$ year/>1 year). Hazard ratios (HRs) and $95 \%$ confidence intervals (CIs) were reported for each predictor/covariate, with the predictor/covariate considered statistically significant if the 95\% CIs did not overlap the null effect of 1 .

The same set of potential predictors use were included as in the analysis of predictors of UC-related hospitalizations, and incidence rate ratios and 95\% CIs reported for each predictor, with statistical significance again indicated by $95 \%$ CIs not overlapping 1. Such predictors for patients experiencing a UC-related hospitalization within the 2-year follow-up period were examined using a negative binomial regression model for nonnegative count estimates [33]. All analyses were performed using Stata, version 15.1 or later [34].

\section{Results}

\section{Eligible Patients}

A total of 649 patients were included in the analysis, of whom 265 (41\%) received adalimumab and 384 (59\%) received infliximab as the indexed biologic (shown in Fig. 2).

\section{Demographics and Clinical Characteristics}

Patient demographics and general health based on the CCI were broadly similar between those receiving adalimumab and those receiving infliximab as the indexed biologic (Table 1). Compared with patients receiving infliximab, patients receiving adalimumab had been diagnosed with UC for longer (3.3 and 4.5 years, respectively; $p<$ 0.01 ) (shown in Table 1). 


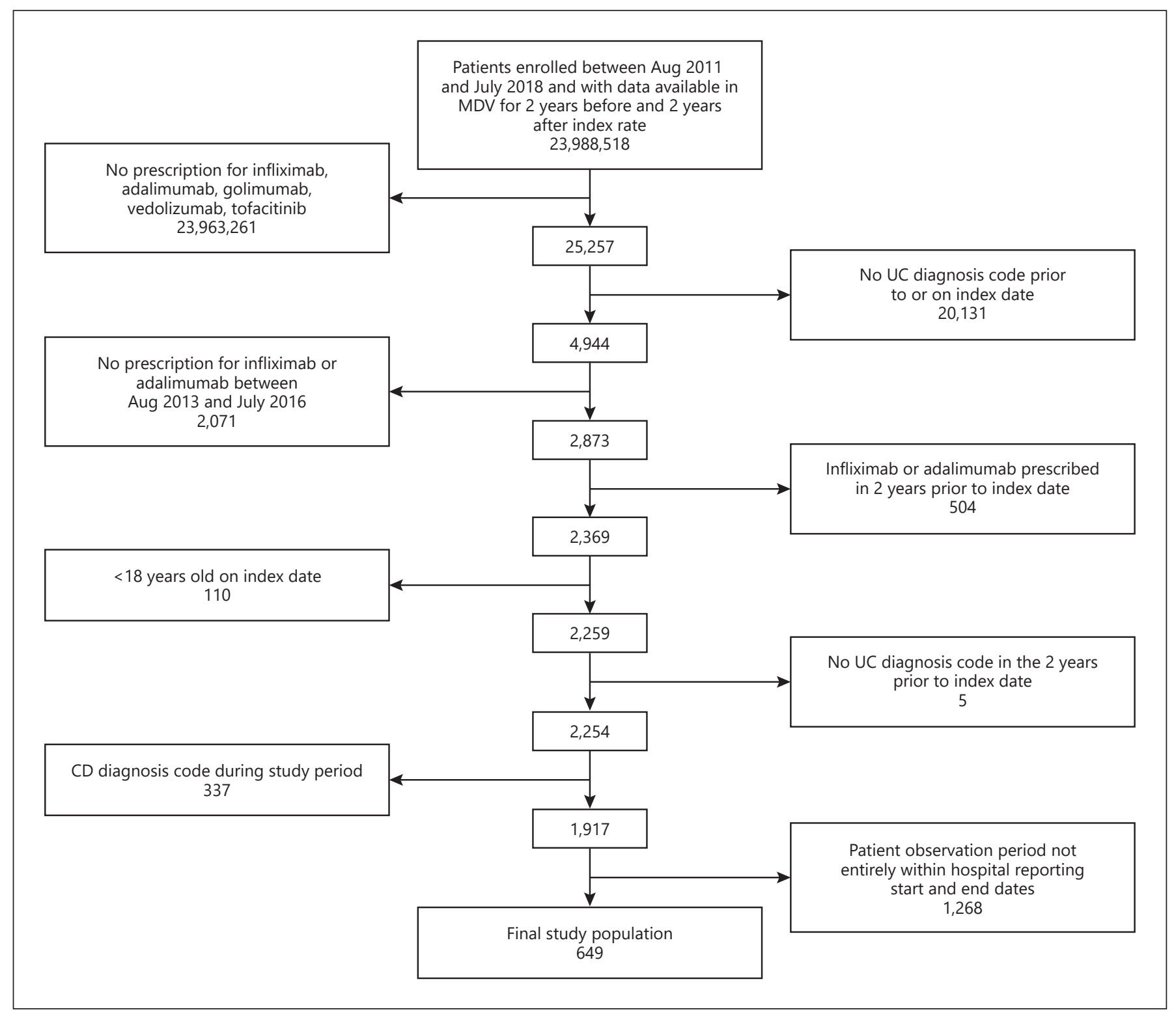

Fig. 2. Consort flow diagram. CD, Crohn's disease; UC, ulcerative colitis.

\section{Primary Objectives}

Adherence and Persistence to Biologic Therapy

Overall, $80 \%$ of patients completed the induction period with the indexed biologic. The proportion of patients completing induction was similar in those receiving adalimumab (82\%) and infliximab (78\%). Adherence to the indexed biologic during the maintenance period was high with both indexed biologics, but patients receiving adalimumab were slightly more adherent than those receiving infliximab, based on PDC (0.95 vs. 0.90$)$; $p<$
0.001) (Fig. 3a). The percentage of patients classified as adherent (PDC $\geq 80 \%$ ) was similar for those receiving adalimumab and infliximab ( $93.6 \%$ vs. $91.0 \%$, respectively, $p=0.33$ ) (shown in Fig. 3).

Based on discontinuation defined as a gap of at least 60 days between refill/infusions, a higher proportion of patients prescribed infliximab on the index data persisted on therapy at $6,12,18$, and 24 months than those prescribed adalimumab (all $p<0.05$ ) (Fig. 3b). Sensitivity analyses using a gap of $\geq 30$ days and $\geq 90$ days between 
Table 1. Patient demographics and clinical characteristics

\begin{tabular}{|c|c|c|c|c|}
\hline & $\begin{array}{l}\text { Total } \\
(n=649)\end{array}$ & $\begin{array}{l}\text { ADA } \\
(n=265)\end{array}$ & $\begin{array}{l}\text { INF } \\
(n=384)\end{array}$ & $p$ value $^{\mathrm{a}}$ \\
\hline \multicolumn{5}{|c|}{ Age at index date, years ${ }^{b}$} \\
\hline Mean (SD) & $43.6(16.1)$ & $44.1(16.2)$ & $43.3(16.0)$ & \multirow{4}{*}{$0.542^{c}$} \\
\hline Min, max & $18.0,90.0$ & $18.0,90.0$ & $18.0,87.0$ & \\
\hline Median & 42 & 42 & 43 & \\
\hline IQR & $30.0,56.0$ & $30.0,56.0$ & $29.0,55.0$ & \\
\hline \multicolumn{5}{|l|}{ Sex, $n(\%)$} \\
\hline Female & $274(42)$ & $120(45)$ & $154(40)$ & \multirow{2}{*}{$0.1966^{d}$} \\
\hline Male & $375(58)$ & $145(55)$ & $230(60)$ & \\
\hline \multicolumn{5}{|c|}{ Time since diagnosis, years } \\
\hline Mean (SD) & $3.8(4.6)$ & $4.5(4.9)$ & $3.3(4.3)$ & \multirow{4}{*}{$0.0019^{c}$} \\
\hline Min, $\max$ & $0.0,26.7$ & $0.0,26.7$ & $0.0,22.9$ & \\
\hline Median & 1.9 & 2.5 & 1.4 & \\
\hline IQR & $0.4,5.9$ & $0.6,7.0$ & $0.4,4.7$ & \\
\hline \multicolumn{5}{|l|}{ Categorized, $n$ (\%) } \\
\hline$\leq 1$ year & $247(38)$ & $84(32)$ & $163(42)$ & \multirow{2}{*}{$0.007^{d}$} \\
\hline$>1$ year & $402(62)$ & $181(68)$ & $221(58)$ & \\
\hline \multicolumn{5}{|l|}{ Baseline CCI } \\
\hline Mean (SD) & $0.7(1.3)$ & $0.8(1.4)$ & $0.7(1.3)$ & \multirow{4}{*}{$0.473^{c}$} \\
\hline Min, max & 0,10 & 0,8 & 0,10 & \\
\hline Median & 0 & 0 & 0 & \\
\hline IQR & 0,1 & 0,1 & 0,1 & \\
\hline \multicolumn{5}{|l|}{ Categorized, $n(\%)$} \\
\hline 0 & $427(66)$ & $173(65)$ & $254(66)$ & \multirow{4}{*}{$0.9155^{f}$} \\
\hline $1-2$ & $168(26)$ & $68(26)$ & $100(26)$ & \\
\hline $3-4$ & $42(7)$ & $18(7)$ & $24(6)$ & \\
\hline $5+$ & $12(2)$ & $6(2)$ & $6(2)$ & \\
\hline \multicolumn{5}{|c|}{ Known baseline extent of disease, $n(\%)^{\mathrm{e}}$} \\
\hline$N$ & 340 & 114 & 226 & \multirow{5}{*}{$0.27^{f}$} \\
\hline Pancolitis & $248(73)$ & $78(68)$ & $170(75)$ & \\
\hline Left-side & $64(19)$ & $28(25)$ & $36(16)$ & \\
\hline Proctosigmoiditis & $16(5)$ & $4(4)$ & $12(5)$ & \\
\hline Proctitis & $12(4)$ & $4(4)$ & $8(4)$ & \\
\hline
\end{tabular}

ADA, adalimumab; CCl, Charlson Comorbidity Index; IFX, infliximab; IQR, interquartile range; SD, standard deviation. ${ }^{a}$ Difference adalimumab versus infliximab. ${ }^{b}$ Maximum age in MDV is 90 years; patients $>90$ years were included as 90 years of age. ' Student's $t$ test.

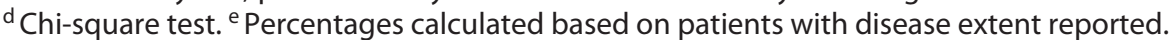
${ }^{f}$ Fisher's exact test; test excludes unknown category (total: 309, ADA: 151, INF: 158). fills/infusions to define discontinuation confirmed these findings.

Health-Care Resource Utilization

The proportion of patients attending outpatient gastroenterology visits and the mean number of visits per patient were numerically higher in the follow-up than the baseline period (Table 2). By contrast, the mean number of UC-related hospitalizations in the follow-up period was numerically lower than that in the baseline period, and lengths of stay were numerically shorter (Table 2). Low numbers of endoscopies and no colectomies were reported during the baseline period and very low numbers of both procedures during the follow-up period, with the reduction in endoscopies presumably reflecting improvements in disease control due to biologic initiation (Table 2). A higher proportion of persistent than nonpersistent patients attended outpatient gastroenterology visits $(p<0.001, p<0.01, p<0.05$ when persistence was assessed at 6,12 , and 18 months, respectively) and the mean number of visits made was higher $(p<$ $0.001, p<0.001, p<0.01$ when persistence was assessed at 6,12 , and 18 months, respectively) for persistent than non-persistent patients (Table 2). However, these visits 

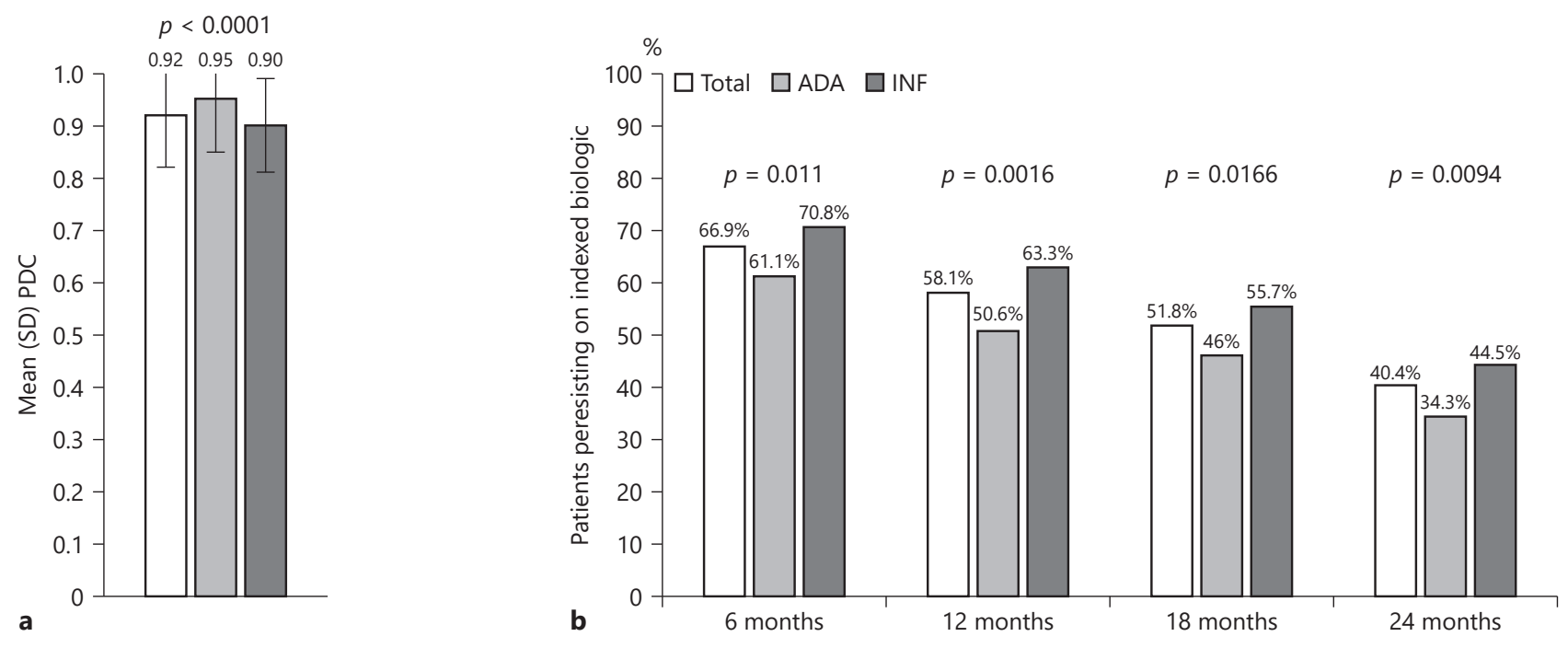

Fig. 3. a Adherence to biologic therapy during the maintenance period. b Persistence to biologic therapy. $p$ values show adalimumab versus infliximab. For a (adherence), Student's $t$ test was used. For $\mathbf{b}$ (persistence), the $\chi^{2}$ test was used (Fisher's exact test used if cell count $<5$ ). ADA, adalimumab; IFX, infliximab; PDC, proportion of days covered; SD, standard deviation.

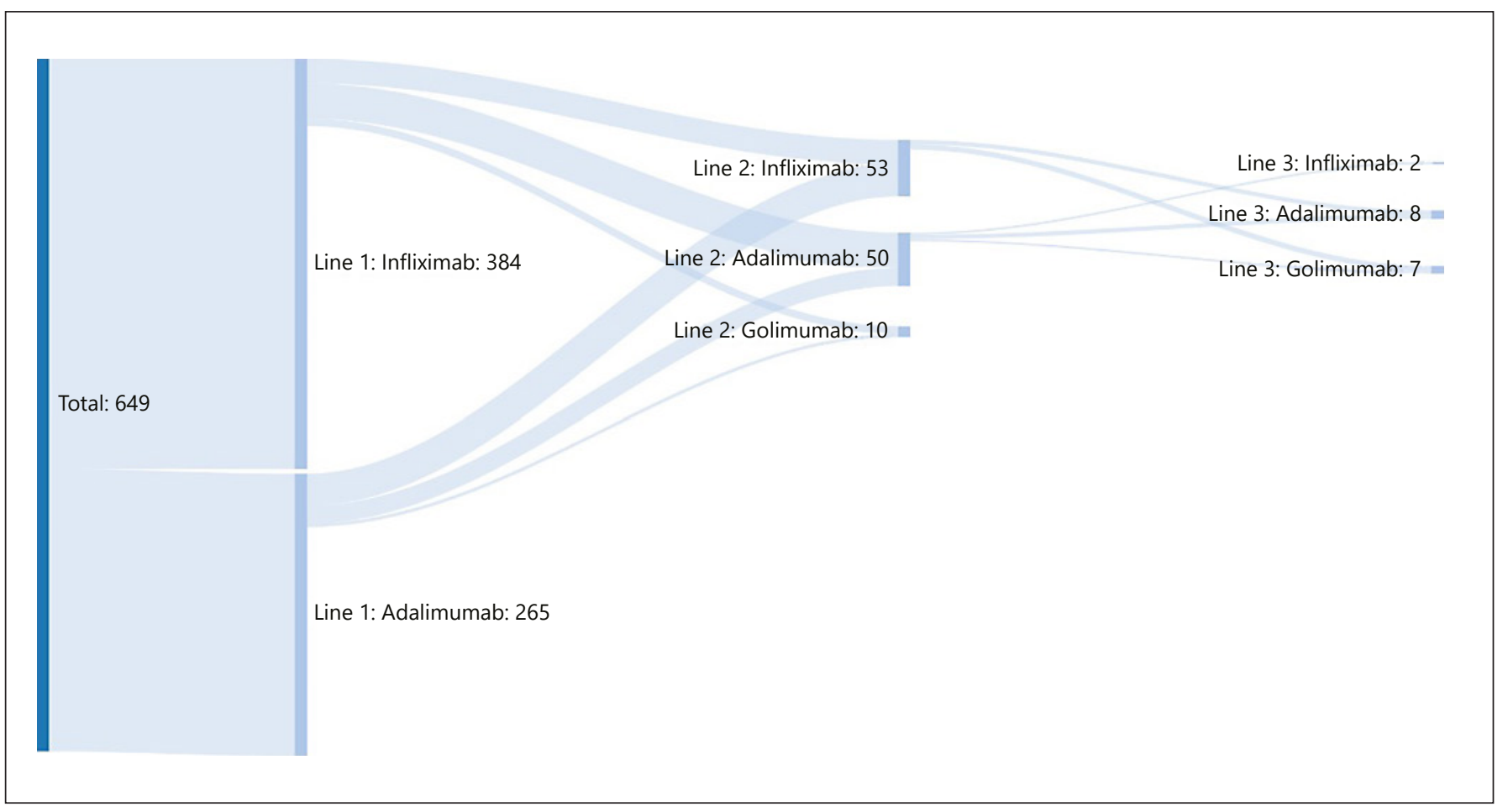

Fig. 4. Biologic use across 1st, 2nd, and 3rd line. ADA, adalimumab; IFX, infliximab. 
include visits for patients to receive infliximab infusions, which resulted in increased visits in persistent versus nonpersistent patients. The mean numbers of UC-related hospitalizations and endoscopies were lower, and hospital stays were of shorter duration, in persistent versus nonpersistent patients, regardless of the time at which persistence was assessed (Table 2). HCRU was not compared between adherent and nonadherent patients because of the very low numbers of nonadherent patients (shown in Table 2).

\section{Secondary Objectives}

Biologic Cycling

Of the total study population, $17.4 \%(n=113)$ received at least a second-line of biologic therapy (Fig. 4); as only infliximab, adalimumab, and golimumab were considered in this analysis, this indicates $17.4 \%$ of patients switching from one TNFi to another. Of these, $47 \%, 44 \%$, and $9 \%$ received infliximab, adalimumab, and golimum$\mathrm{ab}$, respectively, as second-line therapy. Overall, $2.6 \%$ $(n=17)$ of patients received third-line biologic therapy, with infliximab, adalimumab, and golimumab prescribed for $12 \%, 47 \%$, and $41 \%$ of these patients, respectively.

Table 2. UC-related HCRU in baseline versus follow-up and in persistent versus nonpersistent patients

\begin{tabular}{|c|c|c|c|c|c|c|c|c|}
\hline & \multirow{2}{*}{$\begin{array}{l}\text { Baseline } \\
(n=649)\end{array}$} & \multicolumn{7}{|l|}{ Follow-up } \\
\hline & & $\begin{array}{l}\text { total } \\
(n=649)\end{array}$ & \multicolumn{2}{|l|}{6 months } & \multicolumn{2}{|l|}{12 months } & \multicolumn{2}{|l|}{18 months } \\
\hline \multicolumn{9}{|l|}{ Patients with outpatient } \\
\hline $\begin{array}{l}\text { gastroenterology visit(s), } n(\%) \\
\text { Outpatient gastroenterology visits, }\end{array}$ & $\begin{array}{l}363(56) \\
n\end{array}$ & $397(61)$ & $107(50)$ & $290(67)^{* * *}$ & $148(54)$ & $249(66)^{* *}$ & $177(57)$ & $220(66)^{*}$ \\
\hline Min, $\max$ & 0,59 & 0,77 & 0,77 & 0,68 & 0,77 & 0,68 & 0,77 & 0,68 \\
\hline Median & 2 & 11 & 0 & 15 & 2 & 15 & 2 & 15 \\
\hline $\mathrm{IQR}$ & 0,17 & 0,20 & 0,16 & 0,21 & 0,17 & 0,21 & 0,19 & $0,20.5$ \\
\hline \multicolumn{9}{|l|}{ UC-related hospitalizations, $n$} \\
\hline Mean (SD) & $0.8(0.9)$ & $0.5(0.9)$ & $0.8(1.2)$ & $0.3(0.7)^{* * *}$ & $0.8(1.1)$ & $0.2(0.6)^{* * *}$ & $0.7(1.1)$ & $0.2(0.6)^{* * *}$ \\
\hline Mean (SD) & $23.8(35.0)$ & $10.8(26.7)$ & $19.8(36.6)$ & $6.4(18.7)^{* * *}$ & $19.6(35.6)$ & $4.5(14.8)^{* * *}$ & $18.5(34.9)$ & $3.6(11.9)^{* * *}$ \\
\hline Min, $\max$ & 0,301 & 0,232 & 0,232 & 0,149 & 0,232 & 0,149 & 0,232 & 0,106 \\
\hline Median & 10 & 0 & 0 & 0 & 0 & 0 & 0 & 0 \\
\hline IQR & 0,36 & 0,6 & 0,26 & 0,0 & 0,25 & 0,0 & 0,23 & 0,0 \\
\hline \multicolumn{9}{|l|}{ Endoscopies, $n$} \\
\hline Mean (SD) & $0.8(1.4)$ & $0.4(1.0)$ & $0.5(0.9)$ & $0.4(1.0)^{*}$ & $0.6(1.0)$ & $0.3(0.9)^{* *}$ & $0.6(1.2)$ & $0.3(0.7)^{* * *}$ \\
\hline Min, $\max$ & 0,13 & 0,10 & 0,4 & 0,10 & 0,7 & 0,10 & 0,10 & 0,4 \\
\hline Median & 0 & 0 & 0 & 0 & 0 & 0 & 0 & 0 \\
\hline IQR & 0,1 & 0,1 & 0,1 & 0,0 & 0,1 & 0,0 & 0,1 & 0,0 \\
\hline \multicolumn{9}{|l|}{ Colectomies, $n$} \\
\hline Mean (SD) & $0.0(0.0)$ & $0.0(0.2)$ & $0.0(0.2)$ & $0.0(0.1)$ & $0.0(0.2)$ & $0.0(0.1)^{*}$ & $0.0(0.2)$ & $0.0(0.1)$ \\
\hline Min, $\max$ & 0,0 & 0,1 & 0,1 & 0,1 & 0,1 & 0,1 & 0,1 & 0,1 \\
\hline
\end{tabular}

$\mathrm{IQR}$, interquartile range; $\mathrm{SD}$, standard deviation; $\mathrm{HCRU}$, health-care resource utilization; UC, ulcerative colitis. ${ }^{* * *} p<0.001 .^{* *} p<0.1$. $^{*} p<0.5$ for persistent versus nonpersistent. Student's $t$ test was used for numeric variables. Fisher's exact test was used for patients with/without an outpatient gastroenterology visit.

Fig. 5. a Use of biologics, steroids, and IMs across lines of biologic therapy. b Use of steroids across biologic therapy lines and persistence groups. $\mathbf{c}$ Use of IMs across biologic therapy lines and persistence groups. For a, the $\chi^{2}$ test was used. For $\mathbf{b}$ and $\mathbf{c}$ Fisher's exact test was used.

(For figure see next page.) 


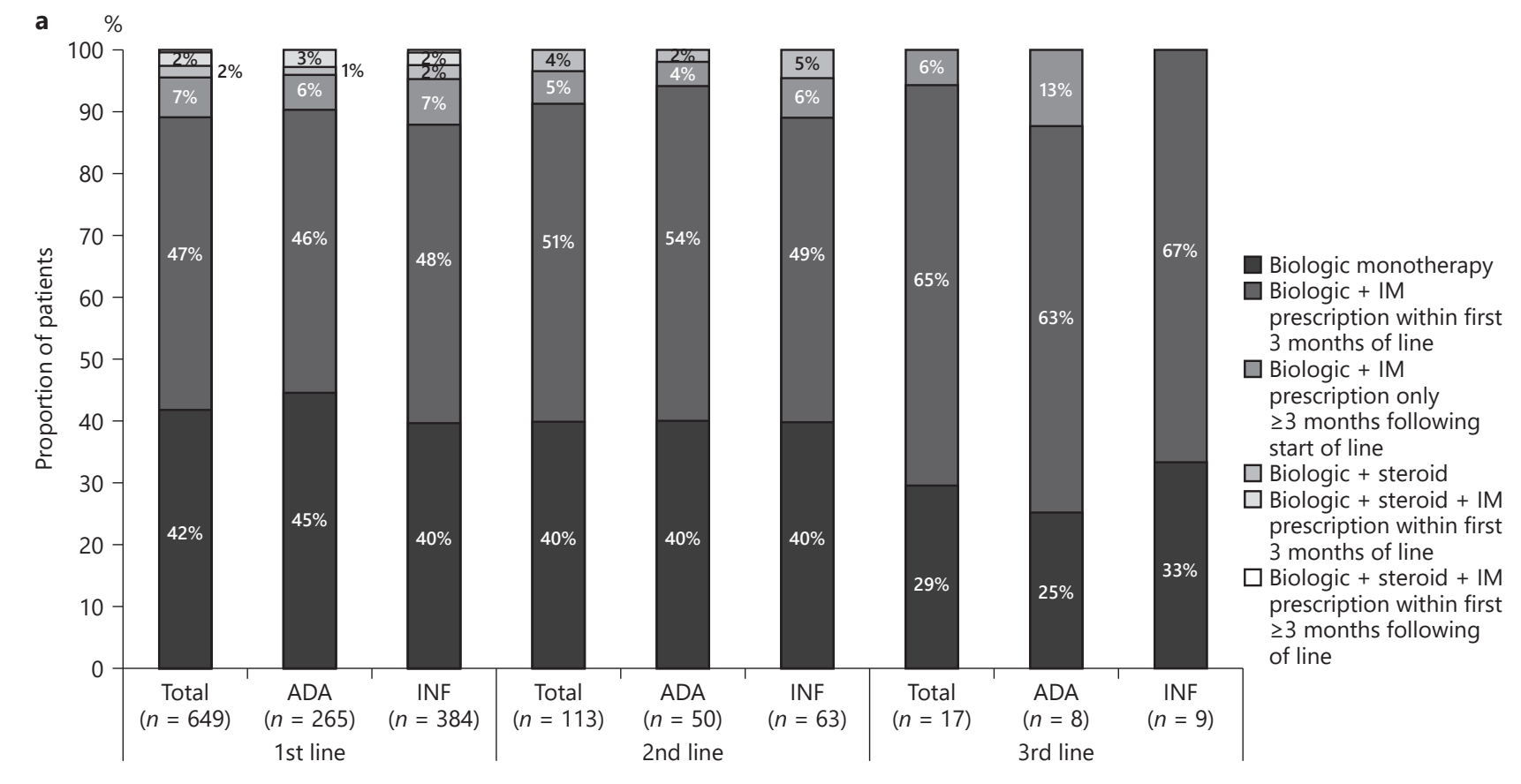

b $\% \square$ Total $\square$ ADA $\square$ INF
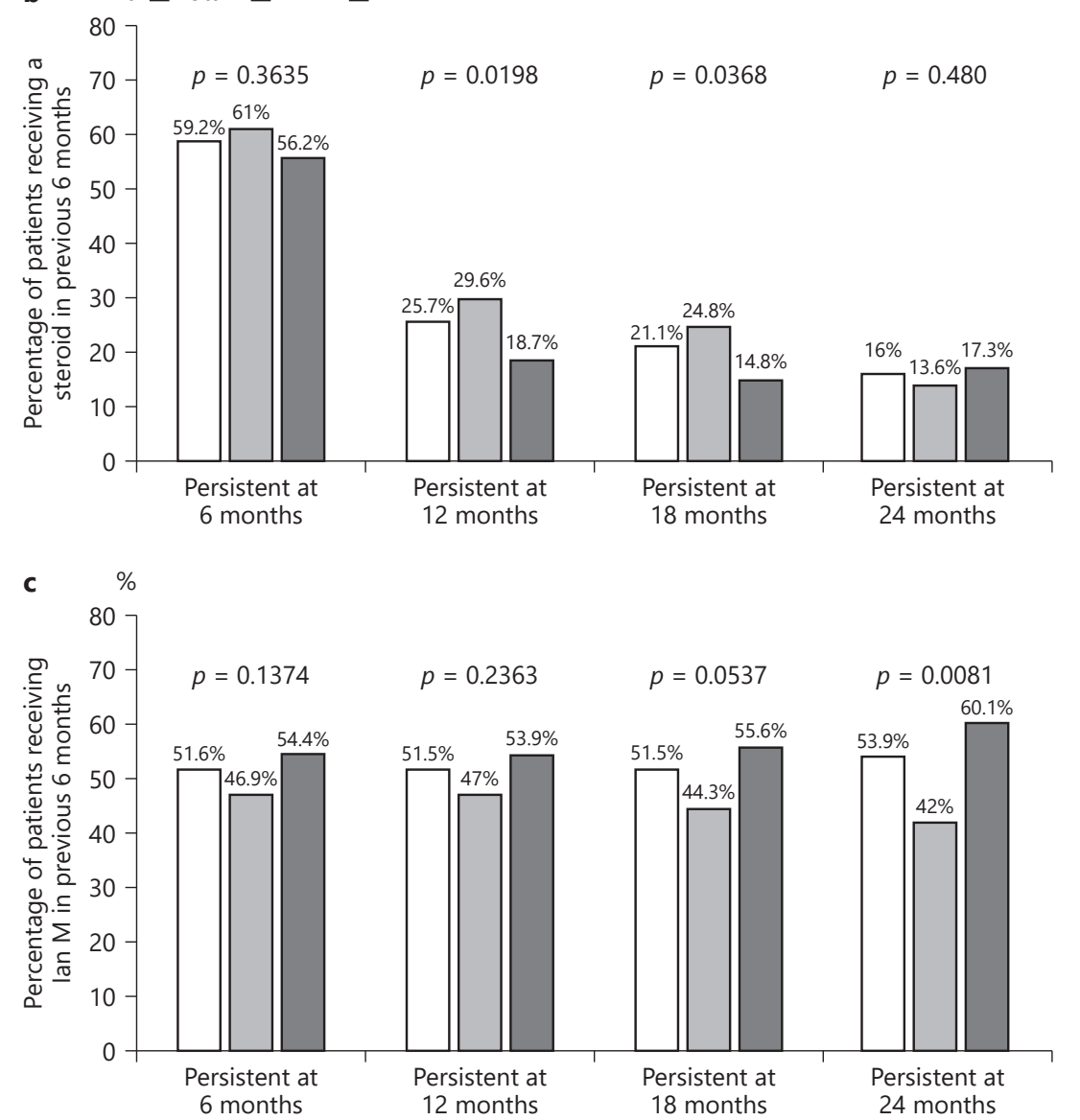


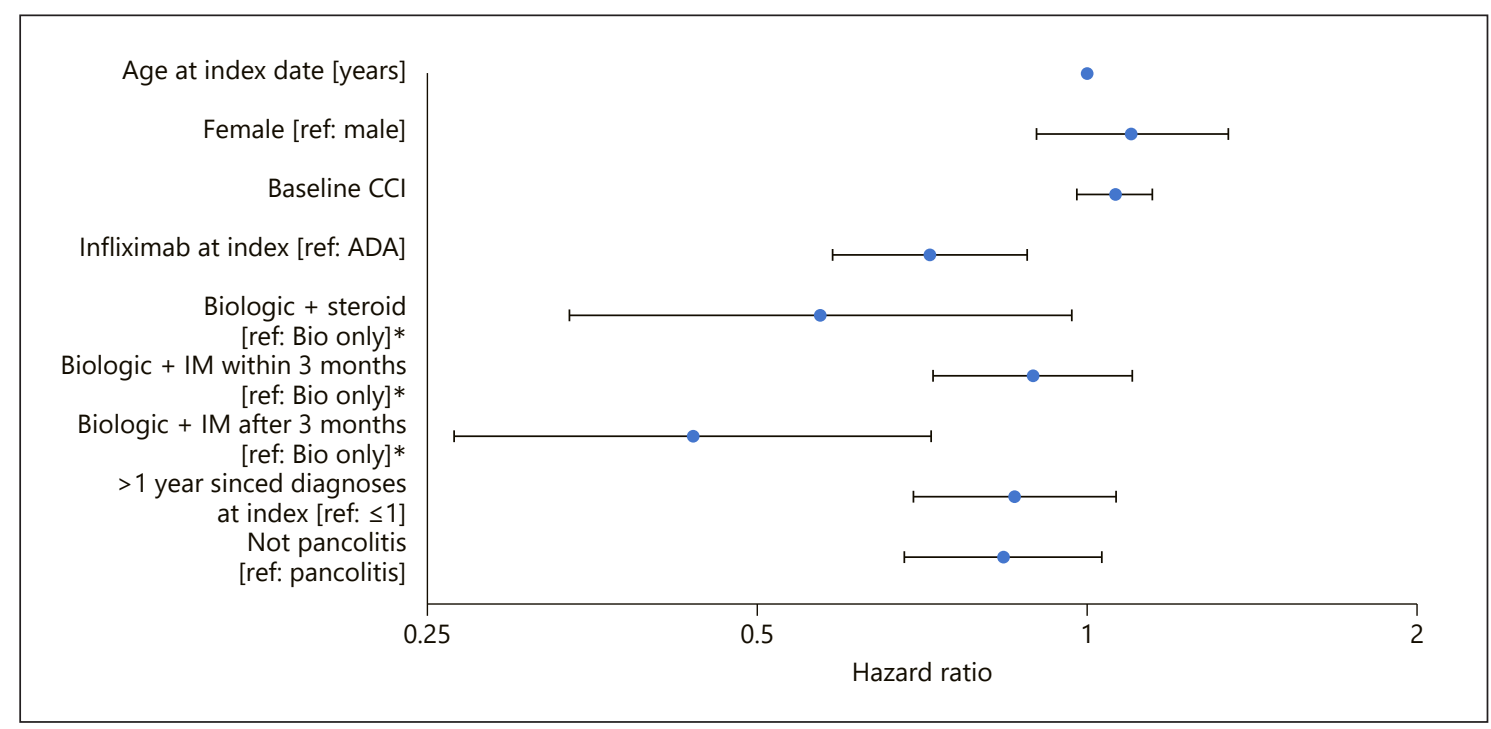

Fig. 6. Predictors of indexed biologic nonpersistence ${ }^{\mathrm{a}}$. ${ }^{\mathrm{a} C o x}$ proportional hazards model of persistence to biology therapy. * Indexed therapy. ADA, adalimumab; Bio, biologic; CCI, Charlson Comorbidity Index; IM, immunosuppressant.

Use of Steroids and IMs

Approximately half of all patients received a biologic in combination with an IM at first, second, and third line (Fig. 5a), the majority of whom received an IM prescription within 3 months of initiating that line of biologic therapy. Less than $5 \%$ of patients received a steroid prescription during a line of biologic therapy (when discounting steroid tapering). There were no significant differences in patterns of concomitant use of steroids and use of IMs between patients receiving adalimumab and those receiving infliximab at any line of therapy (Fig. 5a).

Among patients who persisted with indexed biologic therapy, the use of steroids during biologic therapy in the 6 months prior to assessment of persistence decreased over time (Fig. 5b). Among those persistent at 12 and 18 months, steroid use was significantly lower among those indexed on adalimumab than among those indexed on infliximab. IM use in the 6 months prior to assessment of biologic persistence remained at $\sim 50 \%$ in patients who persisted with indexed biologic therapy at $6,12,18$, and 24 months (shown in Fig. 5c).

Predictors of Nonpersistence with Biologic Therapy

Age, sex, and baseline CCI had no observable effect on the likelihood of patients persisting with biologic therapy (Fig. 6). Patients prescribed infliximab as their indexed therapy were statistically $(p<0.05)$ more likely to remain persistent than those initiated on adalimumab (Fig. 6).
Patients taking biologic therapy in combination with steroids (with or without IMs) were more likely to persist with biologic therapy. Among those receiving prescription for IM $>3$ months after initiating their indexed biologics, the statistically significant $(p<0.05)$ HR of nonpersistence was 0.44; among those who received an IM prescription within 3 months of initiating biologic therapy, the HR of nonpersistence was 0.89 , which was not statistically significant. There was no evidence of a difference in persistence between patients diagnosed $\leq 1$ year $/>1$ year prior to index, or between patients with or without pancolitis (shown in Fig. 6).

Factors Associated with UC-Related Hospitalizations

The hospitalization rate was significantly higher among female patients than male patients but did not differ significantly between the indexed biologics (Fig. 7). Patients were more likely to experience hospitalizations when prescribed an IM within 3 months of initiating biologic therapy than when taking biologics as monotherapy (shown in Fig. 7).

\section{Discussion/Conclusion}

In this real-world study in Japanese patients with moderate-to-severe UC, completion of the biologic treatment induction period and adherence to the indexed biologic 


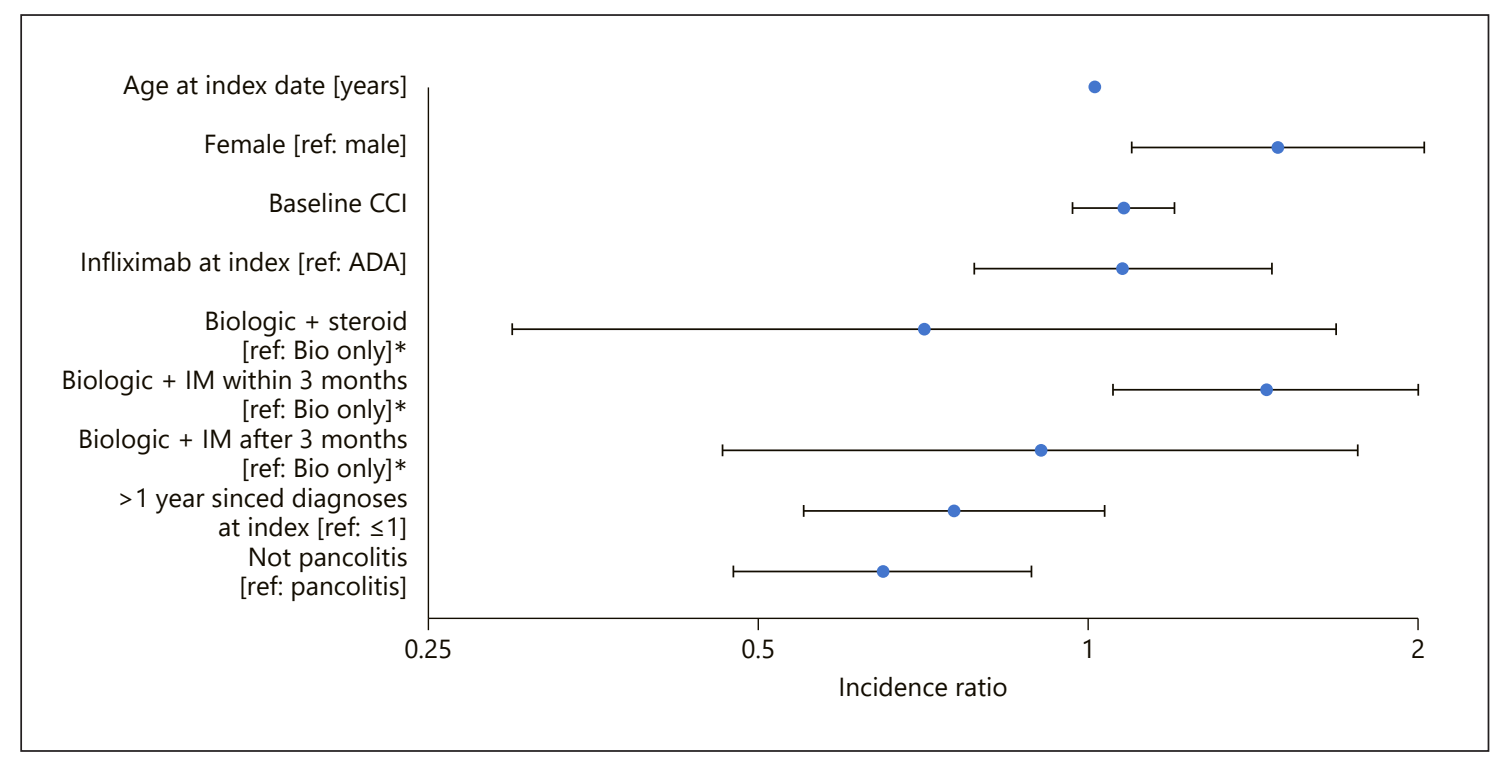

Fig. 7. Predictors of UC-related hospitalizations ${ }^{\mathrm{a} .}{ }^{\mathrm{a}}$ Negative binomial regression model of hospitalizations. *Indexed therapy. ADA, adalimumab; Bio, biologic; CCI, Charlson Comorbidity Index; FU, follow-up period; IM, immunosuppressant; UC, ulcerative colitis.

during the maintenance period were high, but only just over half of patients persisted with the indexed biologic at 18 months, reducing to $40 \%$ at 24 months. Although several studies have reported adherence to biologics to be a problem in patients with inflammatory bowel disease [35-37], in this study, a high level of adherence was observed with both infliximab and adalimumab, and similar to self-reported adherence levels from a survey linked to a US claims database [38], and to levels observed in studies included in a systematic review [37]. High medication adherence levels have been reported in Japan compared with other countries in dialysis patients, possibly as a result of the Japanese health-care system reducing the incidence of cost-related nonadherence [39].

Adherence to adalimumab in the maintenance period was observed to be 0.95 among those indexed on adalimumab and 0.90 among those indexed on infliximab. Higher adherence to infliximab than adalimumab in Crohn's disease but higher adherence to adalimumab than infliximab in RA were reported in a systematic literature review [40].

Patients indexed on an infliximab prescription had been diagnosed with UC for less time than those indexed on adalimumab. This reflects expected care in Japan where infliximab is more frequently used for acute severe UC, which will likely occur earlier in the disease course.

Care for Ulcerative Colitis in Japan
Persistence in our analysis was $67 \%, 58 \%, 52 \%$, and $40 \%$ at $6,12,18$, and 24 months, respectively. This is broadly consistent with the findings from other studies. A claims-based study of patients with UC in South Korea reported 12-month persistence to infliximab and adalimumab of $66.1 \%$ and $62.4 \%$ [41]. A chart review study in South Korea showed that $34.4 \%$ of patients with UC had shown suboptimal response to biologic therapy by 12 months [42]. In the USA, a retrospective claims database cohort study reported 1-year persistence to adalimumab and infliximab of $48 \%$ and $55 \%$ [43], while a retrospective chart review in 14 US community gastroenterology clinics reported $61 \%$ persistence to infliximab at 12 months in biologic-naive patients with UC [44].

Analysis of Japanese claims data reported much higher persistence to biologics in patients with rheumatoid arthritis (RA) who were previously biologic-naive: $99 \%$, $96 \%$, and $95 \%$ at 6,12 , and 18 months, respectively [45]. Persistence beyond 6 months was high between both indexed TNFi medications; $75 \%$ of patients persistent to adalimumab 6 months after index remained persistent at 18 months and $79 \%$ of patients persistent to infliximab 6 months after index remained persistent after 18 months.

In our study, the number of gastroenterology outpatient visits increased from the baseline to the follow-up period, while the number and length of UC-related hospitalizations decreased. These findings suggest that pa- 
tients require closer clinical monitoring but have a reduced need for inpatient care, following initiation of a biologic.

Both the number and length of hospital stays were lower in persistent than nonpersistent patients; if hospitalizations are taken as a proxy for treatment effectiveness, these findings indicate that biologic persistence improves effectiveness and presumably patient outcomes. A similar finding was reported in a US claims analysis, with significantly fewer hospitalizations observed in patients with UC with good adherence to infliximab maintenance therapy than those with poor adherence [46].

In our analysis, $17 \%$ of patients switched to another TNFi as second-line treatment, with $9 \%$ of patients who initiated treatment with infliximab switching to adalimumab, in contrast to a US retrospective claims database cohort study of patients with UC in which $31 \%$ of patients who initiated treatment with infliximab switched to adalimumab [43]. Two studies in patients with RA have reported that patients switching from a TNFi to an agent with an alternative mode of action had significantly higher treatment persistence than those who cycled between TNFi agents [47, 48].

Approximately half of patients used an IM with a TNFi in follow-up, slightly higher than the $41 \%$ of patients who used an IM with biologic therapy in an equivalent study of German patients [28]. Steroid use with a TNFi was substantially lower $(<5 \%)$ than was observed among German patients when accounting for steroid tapering (25\%) [28].

Our study found patients were more likely to persist with biologic therapy if they received infliximab, rather than adalimumab, if they were prescribed a steroid at the same time a TNFi (with or without concomitant IM), and if they received IM therapy $>3$ months after initiating biologic therapy. Higher persistence among those who received concomitant steroid prescriptions may in part result from a group of steroid-dependent patients who initiate biologic therapy as part of a steroid withdrawal strategy and continue therapy with TNFi as a long-term alternative to steroid therapy. In another study of TNFi persistence among Japanese patients with UC, persistence was higher when an IM was taken with infliximab than when on monotherapy; the same trend was not observed among patients who received adalimumab [23]. Concomitant use of 5-ASA was associated with higher persistence of first-line TNFi treatment in patients with $\mathrm{UC}$ in a retrospective cohort analysis of a single European center [49].

Patients who started IM $>3$ months after biologic initiation, likely due to poor response to treatment, were ob- served to be more persistent than those who started IM within 3 months of their biologics. It is expected that patients who started biologics as monotherapy are likely to have less severe UC than those who started biologics alongside an IM. Furthermore, patients who initiated IM $>3$ months after biologic may have overcome the secondary loss of response by the addition of IM, resulting in greater persistence to biologics.

This study has several limitations. The identification of patients with UC via the use of ICD-10 diagnosis codes, with potential for miscoding, might have led to inappropriate inclusion or exclusion of patients from the analysis, and as patients were identified as having moderate-tosevere UC only by initiation of biologic therapy, patients with less severe disease may have been included, although the cost of biologics ensures a very low level of inappropriate prescribing. Although patients using biologics in the 2-year baseline period prior to initiation of a biologic were excluded from this analysis, patients may have received biologic therapy $>2$ years prior to index. It would be unusual however for a biologic to be prescribed after a break of $>2$ years.

While the MDV database is large and is considered largely generalizable to the wider Japan population, the sample only includes patients treated at acute care hospitals. It should be noted however that secondary care takes a highly prominent role in Japanese health care and that there is no system of general practitioners. It is also possible that patients were discharged back to their local units for management after initiating a biologic and might be designated as nonpersistent with decreased outpatient appointments when they were persistent to therapy and attending regular outpatient clinics in a local unit, rather than an acute care hospital. MDV have published results to show that the proportion of patients by age category and major disease category is largely similar between MDV and Ministry of Health, Labour and Welfare of Japan data [50]. It is therefore expected that the UC patient population in MDV will be sufficiently representative of the wider Japanese UC patient population.

Calculation of adherence used the amount of biologic prescribed and recommended dose to estimate the days' medication supply. This approach could lead to an overestimate of adalimumab adherence as prescribed drugs might not necessarily have been taken; a cross-sectional survey of Japanese prescription data suggested that universal public health insurance and relatively low co-payments in Japan result in patients with chronic diseases consulting their physician regularly, and having drugs prescribed depending on the consultation schedule, with 
potential storage of unused drugs [51]. To derive days' supply/coverage, the ATC/DDD system was used for adalimumab, and the recommended dose for a $70-\mathrm{kg}$ patient was used for infliximab; estimated doses may differ from the actual dose and/or duration prescribed to, and taken by, the patient, particularly where individual characteristics are used to determine individual therapeutic doses. Because of a lack of data on dosages prescribed, we were also not able to observe whether a patient had experienced a dosage escalation.

Our analysis did not include any controls for proxies of disease severity as standard proxies are not recorded in the data. Due to the delayed launch of various biologics in Japan, only adalimumab and infliximab could be observed.

It may be concluded that persistence to biologics in Japanese patients with moderate-to-severe UC was poor, and poor persistence was associated with increased nonmedication-associated HCRU, reflecting suboptimal patient outcomes, and resulting in increased burden on the Japanese health-care system. Further research is needed to identify novel treatments for UC that are efficacious, are not associated with substantial HCRU, and that will have high levels of persistence.

\section{Acknowledgment}

Medical writing support under the guidance of the authors was provided by Carole Evans, $\mathrm{PhD}$, on behalf of Adelphi Real World, and was funded by Pfizer Inc, in accordance with Good Publication Practice (GPP3) guidelines (Ann Intern Med 2015; 163:461-464).

\section{Statement of Ethics}

For this study, we used de-identified data provided by a commercial database provider: Medical Data Vision, Co., Ltd. No personal information was provided or could be revealed from the de- identified data. For this reason, no ethical restrictions are imposed on data access by researchers, and ethical approval was not required in accordance with local/national guidelines.

\section{Conflict of Interest Statement}

Joseph C. Cappelleri, Irene Modesto, Marco DiBonaventura, Timothy W. Smith, Sean Gardiner, and Irene Modesto are stockholders and employees of Pfizer Inc (who funded this study). At the time of conducting the study, Danielle Bargo and Takanori Tsuchiya were employees of Pfizer Inc. Theo Tritton and Tim Holbrook are employed by Adelphi Real World, which received funding from Pfizer in connection with the development of this manuscript. At the time of conducting the study, Daniel Bluff was an employee of Adelphi Real World.

\section{Funding Sources}

The study was funded by Pfizer Inc.

\section{Author Contributions}

All authors contributed to conception and design, have been involved in drafting the manuscript and revising it for critically important intellectual content, have given final approval of the version to be published, and agreed to be accountable for all aspects of the work.

\section{Data Availability Statement}

The authors had access to the data for the purposes of this work. Data were accessed through an existing Pfizer license to address the prespecified research questions only. Due to data licensing agreements, the data are not available to include in the submission. The dataset may be obtained on application to MDV: https:// en.mdv.co.jp/application/contactus/form.php.

\section{References}

1 Ungaro R, Mehandru S, Allen PB, Peyrin-Biroulet L, Colombel JF. Ulcerative colitis. Lancet. 2017;389(10080):1756-70.

2 Antonelli E, Villanacci V, Bassotti G. Novel oral-targeted therapies for mucosal healing in ulcerative colitis. World J Gastroenterol. 2018;24(47):5322-30.

3 Satsangi J, Silverberg M, Vermeire S, Colombel JJG. The Montreal classification of inflammatory bowel disease: controversies, consensus, and implications. Gut. 2006;55(6):74953.
4 Ordas I, Eckmann L, Talamini M, Baumgart DC, Sandborn WJ. Ulcerative colitis. Lancet. 2012;380(9853):1606-19.

5 Feuerstein JD, Cheifetz AS. Ulcerative colitis: epidemiology, diagnosis, and management. Mayo Clin Proc; 2014;89(11):1553-63.

6 Ng SC, Shi HY, Hamidi N, Underwood FE, Tang W, Benchimol EI, et al. Worldwide incidence and prevalence of inflammatory bowel disease in the 21 st century: a systematic review of population-based studies. Lancet. 2017;390(10114):2769-78.
7 Morita N, Toki S, Hirohashi T, Minoda T, Ogawa $\mathrm{K}$, Kono $\mathrm{S}$, et al. Incidence and prevalence of inflammatory bowel disease in Japan: nationwide epidemiological survey during the year 1991. J Gastroenterol. 1995;30 Suppl $8: 1-4$.

8 Asakura K, Nishiwaki Y, Inoue N, Hibi T, Watanabe M, Takebayashi T. Prevalence of ulcerative colitis and Crohn's disease in Japan. J Gastroenterol. 2009;44(7):659-65. 
9 Murakami Y, Nishiwaki Y, Oba MS, Asakura K, Ohfuji S, Fukushima W, et al. Estimated prevalence of ulcerative colitis and Crohn's disease in Japan in 2014: an analysis of a nationwide survey. J Gastroenterol. 2019;54(12):1070-7.

10 Da Silva BC, Lyra AC, Rocha R, Santana GO. Epidemiology, demographic characteristics and prognostic predictors of ulcerative colitis. World J Gastroenterol. 2014;20(28):9458.

11 Danese S, Allez M, Van Bodegraven AA, Dotan I, Gisbert JP, Hart A, et al. Unmet medical needs in ulcerative colitis: an expert group consensus. Dig Dis. 2019;37(4):266-83.

12 Wehkamp J, Stange EF. Recent advances and emerging therapies in the non-surgical management of ulcerative colitis. F1000Res. 2018; 7:F1000 Faculty Rev-1207.

13 Matsuoka K, Kobayashi T, Ueno F, Matsui T, Hirai F, Inoue N, et al. Evidence-based clinical practice guidelines for inflammatory bowel disease. J Gastroenterol. 2018;53(3):305-53.

14 Rubin DT, Ananthakrishnan AN, Siegel CA, Sauer BG, Long MD. ACG clinical guideline: ulcerative colitis in adults. Am J Gastroenterol. 2019;114(3):384-413.

15 Harbord M, Eliakim R, Bettenworth D, Karmiris K, Katsanos K, Kopylov U, et al. Third European evidence-based consensus on diagnosis and management of ulcerative colitis. Part 2: current management. J Crohns Colitis. 2017;11(7):769-84.

16 European Crohn's and Colitis Organisation (ECCO). Treatment algorrithms. 2019. Available from: http://www.e-guide.ecco-ibd.eu/ algorithms.

17 Hibi T, Kamae I, Pinton P, Ursos L, Iwakiri R, Hather G, et al., Network meta-analysis: comparative efficacy of biologics in the treatment of biologic-naive Japanese patients with moderately to severely active ulcerative colitis. Inflamm Bowel Dis. 2019;156(3):S27-8.

18 Kobayashi T, Suzuki Y, Motoya S, Hirai F, Ogata H, Ito H, et al. First trough level of infliximab at week 2 predicts future outcomes of induction therapy in ulcerative colitis-results from a multicenter prospective randomized controlled trial and its post hoc analysis. J Gastroenterol. 2016;51(3):241-51.

19 Pharmaceuticals and Medical Devices Agency [PMDA]. New drugs approved in FY 2016 2019. Available from: http://www.pmda.go. jp/files/000229078.pdf\#page $=2$.

20 Pharmafile. Takeda's Entyvio secures Japanese approval in ulcerative colitis approval in ulcerative colitis. 2018 Jul 2.

21 Park SC, Jeen YT, liver . Current and emerging biologics for ulcerative colitis. Gut Liver. 2015;9(1):18-27.

22 Vermeire S, Gils A, Accossato P, Lula S, Marren A. Immunogenicity of biologics in inflammatory bowel disease. Therap Adv Gastroenterol. 2018;11:1756283X17750355.

23 Kobayashi T, Udagawa E, Uda A, Hibi T, Hisamatsu T. Impact of immunomodulator use on treatment persistence in patients with ulcerative colitis: a claims database analysis. J Gastroenterol Hepatol. 2019;35(2):225-32.
24 Higgins PD, Rubin D, Kaulback K, Schoenfield SP, Kane SV. Systematic review: impact of non-adherence to 5-aminosalicylic acid products on the frequency and cost of ulcerative colitis flares. Aliment Pharmacol Ther. 2009;29(3):247-57.

25 Mitra D, Hodgkins P, Yen L, Davis KL, Cohen RD. Association between oral 5-ASA adherence and health care utilization and costs among patients with active ulcerative colitis. BMC Gastroenterol. 2012;12(1):132.

26 Saokaew S, Sugimoto T, Kamae I, Pratoomsoot C, Chaiyakunapruk N. Healthcare databases in Thailand and Japan: potential sources for health technology assessment research. PLoS One. 2015;10(11):e0141993.

27 MDV. Introducing MDV database 2019. Available from: https://www.mdv.co.jp/solution/pharmaceutical/english/.

28 Dignass A, Waller J, Cappelleri JC, Modesto I, Kisser A, Dietz L, et al. Living with ulcerative colitis in Germany: a retrospective analysis of dose escalation, concomitant treatment use and healthcare costs. J Med Econ. 2020;23(4):415-27.

29 Quan H, Li B, Couris CM, Fushimi K, Graham $P$, Hider $P$, et al. Updating and validating the Charlson comorbidity index and score for risk adjustment in hospital discharge abstracts using data from 6 countries. Am J Epidemiol. 2011;173(6):676-82.

30 Martin BC, Wiley-Exley EK, Richards S, Domino ME, Carey TS, Sleath BL. Contrasting measures of adherence with simple drug use, medication switching, and therapeutic duplication. Ann Pharmacother. 2009;43(1):36-44.

31 Freeman GH, Halton JH. Note on an exact treatment of contingency, goodness of fit and other problems of significance. Biometrika. 1951;38(1/2):141-9.

32 Rosner B. Fundamentals of biostatistics: Nelson Education; 2015.

33 Long JS, Freese J. Regression models for categorical dependent variables using Stata: Stata press; 2006.

34 Stata. Stata statistical software: release 15. College Station, TX: StataCorp LLC; 2017.

35 Chan W, Chen A, Tiao D, Selinger C, Leong $\mathrm{R}$. Medication adherence in inflammatory bowel disease. Intest Res. 2017;15(4):434-45.

36 van der Have M, Oldenburg B, Kaptein AA, Jansen JM, Scheffer RC, van Tuyl BA, et al. Non-adherence to anti-TNF therapy is associated with illness perceptions and clinical outcomes in outpatients with inflammatory bowel disease: results from a prospective multicentre study. J Crohns Colitis. 2016;10(5):549-55.

37 Lopez A, Billioud V, Peyrin-Biroulet C, PeyrinBiroulet L. Adherence to anti-TNF therapy in inflammatory bowel diseases: a systematic review. Inflamm Bowel Dis. 2013;19(7):1528-33.

38 Lofland JH, Johnson PT, Ingham MP, Rosemas SC, White JC, Ellis L, et al. Shared decision-making for biologic treatment of autoimmune disease: influence on adherence, persistence, satisfaction, and health care costs. Patient Prefer Adherence. 2017;11:947-58.
39 Hirth RA, Greer SL, Albert JM, Young EW, Piette JD. Out-of-pocket spending and medication adherence among dialysis patients in twelve countries. Health Aff. 2008;27(1):89-102.

40 Fidder HH, Singendonk MM, van der Have M, Oldenburg B, van Oijen MG. Low rates of adherence for tumor necrosis factor- $\alpha$ inhibitors in Crohn's disease and rheumatoid arthritis: results of a systematic review. World J Gastroenterol. 2013;19(27):4344-50.

41 Jung YS, Han M, Park S, Cheon JH. Biologic use patterns and predictors for non-persistence and switching of biologics in patients with inflammatory bowel disease: a nationwide population-based study. Dig Dis Sci. 2020;65(5):1436-44.

42 Yamamoto-Furusho JK, Al Harbi O, Armuzzi A, Chan W, Ponce de Leon E, Qian J, et al. Incidence of suboptimal response to tumor necrosis factor antagonist therapy in inflammatory bowel disease in newly industrialised countries: The EXPLORE study. Dig Liver Dis. 2020;52(8):869-77.

43 Null KD, Xu Y, Pasquale MK, Su C, Marren A, Harnett J, et al. Ulcerative colitis treatment patterns and cost of care. Value Health. 2017; 20(6):752-61.

44 Waters H, Vanderpoel J, McKenzie S, Lunacsek O, Franklin M, Lennert B, et al. Stability of infliximab dosing in inflammatory bowel disease: results from a multicenter US chart review. J Med Econ. 2011;14(4):397-402.

45 Mahlich J, Sruamsiri R. Persistence with biologic agents for the treatment of rheumatoid arthritis in Japan. Patient preference Adherence. 2016;10:1509-19.

46 Carter CT, Leher H, Smith P, Smith DB, Waters HC. Impact of persistence with infliximab on hospitalizations in ulcerative colitis. Am J Manag Care. 2011;17(6):385-92.

47 Chastek B, Chen CI, Proudfoot C, Shinde S, Kuznik A, Wei W. Treatment persistence and healthcare costs among patients with rheumatoid arthritis changing biologics in the USA. Adv Ther. 2017;34(11):2422-35.

48 Bonafede MMK, McMorrow D, Proudfoot C, Shinde S, Kuznik A, Chen CI, et al. Treatment persistence and healthcare costs among patients with rheumatoid arthritis after a change in targeted therapy. Am Health Drug Benefits. 2018;11(4):192-202.

49 Pouillon L, Baumann C, Rousseau H, Choukour M, Andrianjafy C, Danese S, et al. Treatment persistence of infliximab versus adalimumab in ulcerative colitis: a 16-year singlecenter experience. Inflamm Bowel Dis. 2019; 25(5):945-54.

50 Medical Data Vision (MDV). Comparison of the number of patients in the MDV database with MHLW data. MDV Mail Magazine; 2016.

51 Koyanagi K, Kubota T, Kobayashi D, Kihara T, Yoshida T, Miisho T, et al. Prescription factors associated with medication non-adherence in Japan assessed from leftover drugs in the SETSUYAKU-BAG campaign: focus on oral antidiabetic drugs. Front Pharmacol. 2016;7:212. 